\title{
Experimental evidence for semiconducting behavior of Si-XII
}

\author{
S. Ruffell, ${ }^{1, *}$ K. Sears, ${ }^{1}$ A. P. Knights, ${ }^{2}$ J. E. Bradby, ${ }^{1}$ and J. S. Williams ${ }^{1}$ \\ ${ }^{1}$ Department of Electronic Materials Engineering, Research School of Physics \& Engineering, \\ Australian National University, Canberra, 0200, Australia \\ ${ }^{2}$ Department of Engineering Physics, McMaster University, 1280 Main Street West, Hamilton, Ontario, L8S 4L7, Canada
}

(Received 17 November 2010; published 25 February 2011)

\begin{abstract}
The conventional diamond cubic phase of silicon, so-called Si-I, exhibits the desirable semiconducting properties on which the silicon chip industry relies and it is by far the most stable of all silicon phases. However, other phases of silicon can be formed under pressure and some of them are metastable at room temperature and pressure. Two such phases, Si-III (BC8) and Si-XII (R8), can be produced by indentation with a diamond tip but, despite an understanding of their structure, little is known about their electrical properties. As we demonstrate experimentally, such phases can have entirely different (electrical) properties to normal (diamond cubic) silicon, consistent with recent theoretical studies that predict Si-XII to be a narrow-band-gap semiconductor and Si-III to be a semimetal. We report here electrical measurements on the Si-XII phase and demonstrate that it is indeed a semiconductor. Furthermore, and somewhat surprisingly, both boron and phosphorus can be electrically activated in the Si-XII structure during its formation by indentation at room temperature.
\end{abstract}

DOI: 10.1103/PhysRevB.83.075316

PACS number(s): 72.80.Cw, 73.40.Lq, 73.61.Cw, 81.05.Zx

\section{INTRODUCTION}

Diamond cubic silicon can transform into several different phases under the application of pressure and some phases are stable at room temperature and pressure. Two such crystalline phases are so-called Si-XII (R8) (Ref. 1) and Si-III $(\mathrm{BC} 8){ }^{2}$ Whereas high-pressure diamond-anvil experiments result almost entirely in the $\mathrm{Si}$-III end phase (and hence few measurements of properties of Si-XII exist), nanoindentation allows highly controlled formation of zones of mixed Si-III and $\mathrm{Si}$-XII phases down to nanometer dimensions. ${ }^{3}$ In particular, during loading at room temperature to a pressure $<11 \mathrm{GPa}$, the crystalline silicon $(c-\mathrm{Si})$ phase transforms to the metallic phase, Si-II. This phase is unstable at pressures below 8 $\mathrm{GPa}$, and further phase transforms during unloading to either amorphous silicon $(a-\mathrm{Si})$ or a mixture of high-pressure polycrystalline phases, so-called Si-III and Si-XII, depending on the experimental conditions. Whereby slow unloading favors crystalline phases and fast unloading favors the formation of $a$ $\mathrm{Si}^{4-10}$ In addition, relaxed ${ }^{11}$ ion-implanted amorphous silicon has been found to undergo similar phase transformations, thus allowing formation of residual high-pressure crystalline phases within an $a$-Si matrix..$^{10,12,13}$ Furthermore, the dominant end phase following nanoindentation is $\mathrm{Si}$-XII, although this phase is mixed with up to $\sim 20 \% \mathrm{Si}$-III in a polycrystalline residual zone. $^{3}$

Apart from structural information, little is known about the electrical properties of Si-III and Si-XII. For example, an early experimental study in a high-pressure (diamond-anvil) cell indicated that $\mathrm{Si}$-III was a semimetal, ${ }^{14}$ but no electrical measurements have been made on Si-XII. In addition, theoretical studies variously predict that $\mathrm{Si}$-XII is either a semimetal or a narrow-band-gap semiconductor. ${ }^{15-17}$ In this study we have formed mixed phases of $\mathrm{Si}-\mathrm{III}$ and $\mathrm{Si}-\mathrm{XII}$ in amorphous silicon by indentation pressure using a sharp diamond indenter. The electrical properties of these phases are subsequently investigated using both through-wafer probing with a conducting diamond tip in the indentation apparatus and test structures to measure electrical conductivity and carrier mobility following indentation. The results provide the experimental measurements of the electrical properties of mixed $\mathrm{Si}$-III and $\mathrm{Si}$-XII phases of silicon created by nanoindentation. We demonstrate that the $\mathrm{Si}$-XII phase of $\mathrm{Si}$ is a semiconductor, but with electrical behavior that is very different from conventional diamond cubic silicon, including the ability to dope it both $n$ and $p$ type during the indentation process at room temperature without the need for an annealing step.

\section{EXPERIMENT}

We used three different sample structures to measure the electrical properties of zones of Si-III and Si-XII created by indentation. In each case, the indentation conditions (see below) were carefully chosen so as to form continuous regions of mixed Si-III and SI-XII phases as confirmed using Raman spectroscopy and cross-sectional transmission electron microscopy (XTEM). Raman spectroscopy was performed using a Renishaw 2000 instrument fitted with a 632-nm laser that could be focused to a spot size of $\sim 1 \mu \mathrm{m}$ diameter. A focused ion-beam milling and pluck-out method was used for preparation of XTEM samples. Analysis of the samples was performed using a Philips CM 300 electron microscope.

The first electrical method [Fig. 1(a)] uses a bulk Czochralski-grown crystalline $\mathrm{Si}(100)$ wafer (low-level background doping of boron at $\sim 10^{15} \mathrm{~cm}^{-3}$ ) and is referred to as the "through-tip" method. This method probes the conductivity in the vertical direction through a single indent via a conducting (boron-doped) indenter tip. Further details on the in situ electrical measurement system can be found elsewhere. ${ }^{18,19}$ In this method, an entire surface layer of $a$-Si of $\sim 50 \mathrm{~nm}$ was created by $50-\mathrm{keV} \mathrm{Si}$ ion implantation and then relaxed by annealing at $450{ }^{\circ} \mathrm{C}$ for $30 \mathrm{~min}$. The boron sheet concentration in the $a$-Si layer was modified by $\mathrm{B}$ ion implantation at $20 \mathrm{keV}$ to fluences of $10^{14}$ and $10^{15} \mathrm{~cm}^{-2}$. This results in peak boron concentrations of $\sim 10^{19}$ 
(a)

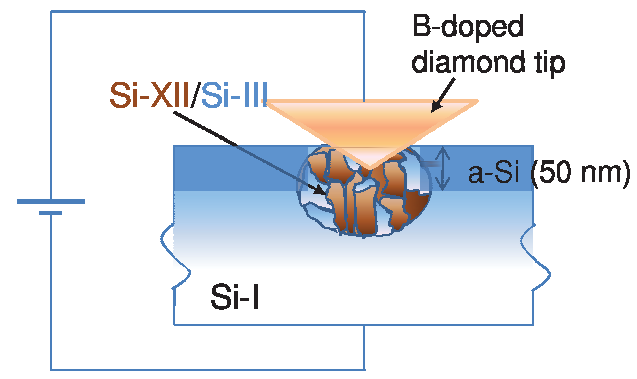

(b)

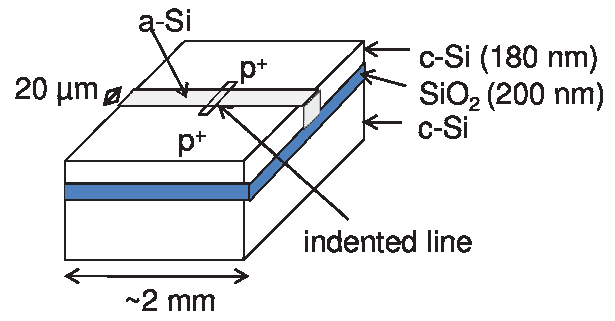

FIG. 1. (Color online) Schematics of the two types of electrical measurements to assess the conductivity of mixed zones of Si-III and Si-XII. The "through-tip" technique is shown in (a). Here a conductive indenter tip is in contact with the surface of the phase-transformed silicon and the current flow through the sample is measured as a function of voltage applied across the sample and the indenter tip. In the "resistive bar" structure (b), $I-V$ measurements are made between two heavily doped contact pads that are electrically connected by a bar of $\mathrm{Si}$-III and Si-XII across an electrically insulating strip of $a$-Si. The bar is formed by a series of overlapping indents.

and $\sim 10^{20} \mathrm{~cm}^{-3}$, respectively, at a depth of $80 \mathrm{~nm}$. The $a-\mathrm{Si}$ is an electrically insulating layer between the surface and the underlying crystalline wafer. Indentation was performed using a Hysitron Triboindenter fitted with a conducting diamond Berkovich tip with a maximum load of $10 \mathrm{mN}$. These loading conditions result in a zone of Si-III and Si-XII, which punches through this insulating layer into the underlying $\mathrm{Si}$-I substrate to a depth of $\sim 250 \mathrm{~nm}$. The conductivity of the indented region could therefore be probed by measuring the resistivity from the top surface of the indent to the semiconductor substrate, before and after indentation.

The second structure [Fig. 1(b)] is fabricated on a siliconon-insulator (SOI) wafer and is referred to as the "resistive bar" structure. The SOI wafer consists of a 180-nm crystalline $\mathrm{Si}(100)$ layer (low-level background doping of boron at $\sim 10^{15} \mathrm{~cm}^{-3}$ ) on top of a 200-nm layer of $\mathrm{SiO}_{2}$ on a crystalline silicon substrate. A $20-\mu \mathrm{m}$-wide strip of amorphous silicon ( $a$-Si) was formed across the SOI wafer by $\mathrm{Si}$ ion implantation and then relaxed by annealing at $450{ }^{\circ} \mathrm{C}$ for $30 \mathrm{~min}$. This $a$-Si strip extends down to the $\mathrm{SiO}_{2}$ layer and electrically isolates the two sides of the sample, which act as contact pads. The crystalline Si material on either side of the $a$-Si strip was highly doped with boron to facilitate Ohmic contacts to these pads so that direct resistance measurements could be made across the $a$-Si strip both before and after indentation using an $I-V$ probe station. As with the "through-tip" sample structures, B ion implantation at an energy of $20 \mathrm{keV}$ was used to control the sheet concentration in the $a$-Si strip. A series of overlapping indents was then made across the $a$-Si strip to form a continuous line of high-pressure phase material to electrically connect the two sides. The indent lines were made using an Ultra MicroIndentation system (UMIS) 2000 with a spherical diamond indenter of $\sim 10 \mu \mathrm{m}$ radius. A maximum load of $200 \mathrm{mN}$ was used for each indent, which ensures an average applied pressure of $<11 \mathrm{GPa}$ over a region extending from immediately under the indenter contact to the $\mathrm{SiO}_{2}$ layer. This results in a resistive bar of $\mathrm{Si}$-III and $\mathrm{Si}$-XII with a width of $\sim 4 \mu \mathrm{m}$ and length corresponding to the $a$-Si strip width $(20 \mu \mathrm{m})$. The bar extends $180 \mathrm{~nm}$ in depth to the $\mathrm{SiO}_{2}$, allowing the cross-sectional area of the line to be determined. These structures allow quantitative measurements of resistivity and further investigation of the effect of boron on the conductivity as well as information on the electrical contacts between nanosized grains of Si-III and $\mathrm{Si}$-XII (see the discussion later).

The third method allows resistivity, carrier density, and carrier mobility measurements to be made. Here, unique "micro-Hall" van der Pauw structures were fabricated in the SOI material (Fig. 2). Cloverleaf mesas were fabricated with a central region of $20 \times 20 \mu \mathrm{m}^{2}$. This relatively small size allowed the whole of the region to be transformed to Si-III and Si-XII by performing an array of overlapping indents under the same conditions as those for the resistance bars. Sheet resistivity, carrier density, and carrier mobility measurements could then be made on the phase-transformed material using an Accent HL5500PC Hall-effect measurement system. In addition, this technique allowed electrical measurements to be made on the Si-III and Si-XII zones created in both $c$-Si and $a-\mathrm{Si}$ matrices. The other measurement techniques described here are limited to indentation in $a$-Si.

(a)

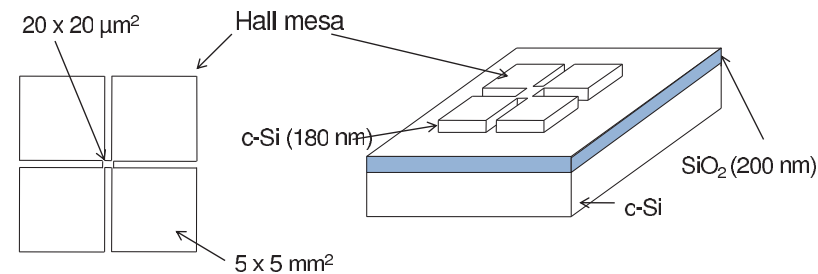

(b)

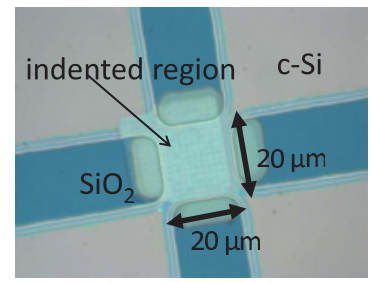

FIG. 2. (Color online) Schematic of the van der Pauw structure fabricated (in SOI) for the micro-Hall measurements is shown in (a). Resistivity and Hall measurements were made on these structures where the central region $\left(20 \times 20 \mu \mathrm{m}^{2}\right)$ was transformed to Si-III and Si-XII by a series of overlapping indents. An optical microscope image of the indented central region is shown in (b). 


\section{RESULTS}

Figure 3 shows $I-V$ characteristics measured using the through-tip apparatus of $\mathrm{Si}$-III and $\mathrm{Si}$-XII zones formed in the $a$-Si containing a range of $\mathrm{B}$ sheet concentrations. The measurements were extracted at the end of the unload cycle. Before indentation, no current flow occurs through the $a$-Si layer. Following room-temperature indentation to form $\mathrm{Si}$-III and $\mathrm{Si}-\mathrm{XII}$, a rectifying $I-V$ characteristic is measured. The rectifying $I-V$ characteristics can originate from the electrical contacts formed between the tip and the high-pressure phases and/or between the high-pressure phases and the underlying crystalline substrate. In forward bias, the current increases with boron content in the $a$-Si film and scales approximately with the sheet boron concentration. This increase in conductivity under forward bias with increasing boron content is suggestive of electrical activation of boron during the phase-transformation process at room temperature. This apparent B "doping" raises the interesting question: Is Si-XII behaving as a semiconductor? To confirm such behavior further, more detailed measurements are required as illustrated below.

Figure 4 shows $I-V$ characteristics measured for a series of resistive bars of $\mathrm{Si}$-III and $\mathrm{Si}-\mathrm{XII}$ containing a range of boron sheet concentrations. The conductivity across the $a$-Si strip increases substantially when a $4-\mu \mathrm{m}$-wide line of Si-III and $\mathrm{Si}-\mathrm{XII}$ is formed to connect the contact pads. Indeed, in the case of the "low boron" starting sample, the resistance (per unit width) measured across the amorphous strip decreases by two orders of magnitude after indenting. As the boron content of the $a$-Si strip prior to indentation is increased, the conductivity of the bar after indentation increases again by an order of magnitude. Note that there is some variation in conductivity for samples without additional boron, as illustrated by the three "low boron" curves in Fig. 4, and is possibly owing to

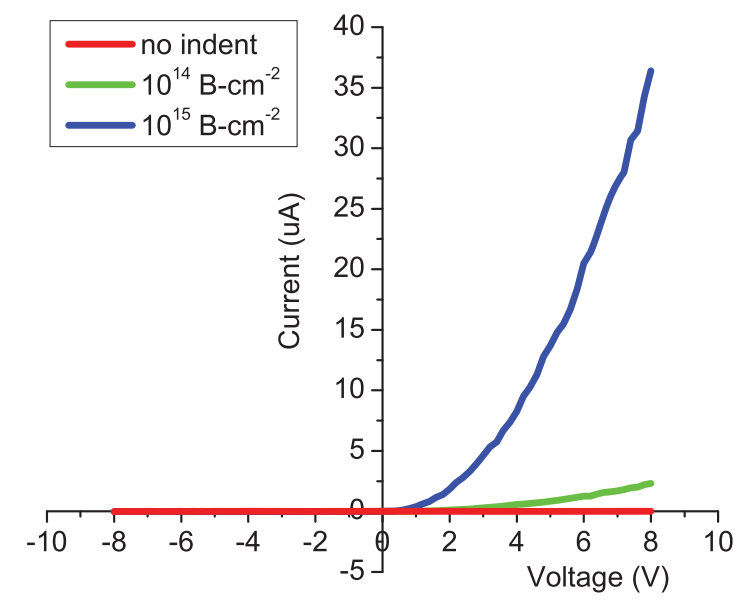

FIG. 3. (Color online) Through-tip $I$ - $V$ characteristics of the Si-III and Si-XII zones formed through a surface $a$-Si layer containing implanted boron (through-tip measurements). Prior to indentation, there is essentially no current flow in either forward or reverse direction, i.e., through the insulating $a$-Si layer. After indentation, the forward-biased current increases dramatically, indicating that the phase-transformed zone has a much higher conductivity compared with $a$-Si. In addition, the forward-biased current scales with the sheet boron concentration.

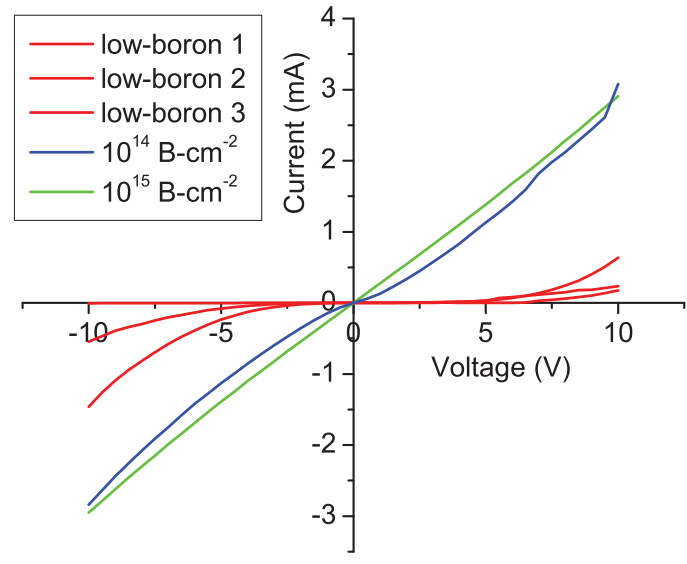

FIG. 4. (Color online) Summary of measurements made on resistance bar structures formed in SOI samples. The $I-V$ characteristics of structures containing only a low (background) concentration of boron ("low boron") and from those containing additional boron are shown. Structures in "low boron" material show nonlinear behaviour and variation across nominally identical samples. The origin of this non-Ohmic behavior is discussed later. The resistivity decreases with increasing boron content and the $I-V$ characteristics show less variation and become more Ohmic.

the random nature of the $\mathrm{Si}$-III and/or $\mathrm{Si}-\mathrm{XII}$ nucleation and growth during indentation pressure release, ${ }^{5,7,10,13,20-25}$ as we explain more fully later. Also note that the $I-V$ characteristics of the samples without additional boron in Fig. 4 do not exhibit good (linear) Ohmic behavior. Furthermore, (i) this non-Ohmic behavior is also observable for the $10^{14} \mathrm{~B} \mathrm{~cm}^{-2}$ samples at low voltages and (ii) the resistance does not scale with boron content for the $10^{14}$ and $10^{15} \mathrm{~B} \mathrm{~cm}^{-2}$ samples, as might have been expected based on the through-tip measurements (Fig. 3). Additionally, for the highest boron content $\left(10^{15} \mathrm{~B} \mathrm{~cm}^{-2}\right)$ the $I-V$ characteristics are very consistent over several samples and exhibit good Ohmic behavior. We believe that these effects relate to the Schottky-like character of contacts between the $\mathrm{Si}$-III and Si-XII domains along the bar, as we discuss more fully later.

The resistance of the Si-III and Si-XII bar can be estimated by applying a linear fit to the $I-V$ curves in Fig. 4, giving resistances of $15-40 \mathrm{k} \Omega$ for the bars without additional implanted boron. This gives a resistivity of $\sim 0.05-0.14 \Omega$ $\mathrm{cm}$ for the Si-III and Si-XII bar, which is interestingly lower than that of the starting $c$-Si wafers $(14-22 \Omega \mathrm{cm})$. Clearly, the $\mathrm{Si}$-III and $\mathrm{Si}-\mathrm{XII}$ regions, even in samples without additional boron, are highly conductive. The conductivity of the Si-III and Si-XII bars can be further increased (by an order of magnitude) by boron implantation prior to indentation, as shown by the $I-V$ curves in Fig. 4. For a sheet concentration of $10^{14} \mathrm{~B} \mathrm{~cm}^{-2}$ the indented line has a resistance of $\sim 4 \mathrm{k} \Omega$. This is decreased further (to $3 \mathrm{k} \Omega$ ) for $10^{15} \mathrm{~B} \mathrm{~cm}^{-2}$. This corresponds to a resistivity of $\sim 0.01 \Omega \mathrm{cm}$, an order of magnitude lower than a bar containing no additional boron $(\sim 0.1 \Omega \mathrm{cm})$.

In order to confirm boron doping of Si-III and Si-XII suggested by the resistivity measurements, micro-Hall measurements were performed to measure carrier-transport properties of the Si-III and Si-XII zones. Figure 5 summarizes the Hall measurements made on Si-III and Si-XII patterns. Also shown 


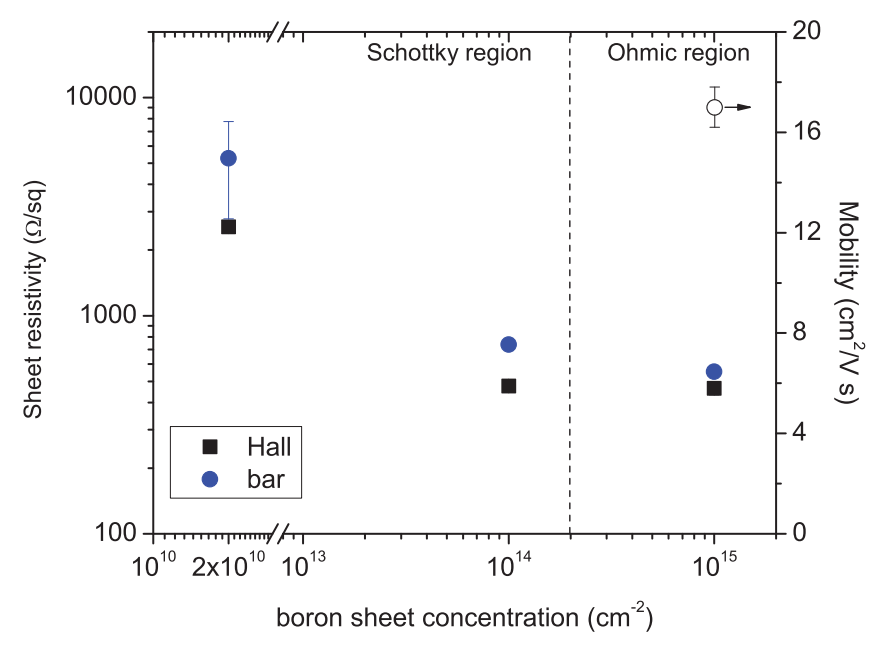

FIG. 5. (Color online) This figure summarizes the Hall measurements on zones of Si-III and Si-XII formed in $a$-Si as a function of sheet boron concentration. Also included are the resistance bar measurements for comparison. An estimate of the boron sheet concentration required to overcome Schottky-like barriers between grains of Si-III and Si-XII (i.e., for the whole zone to exhibit Ohmic behavior) is shown. This divides the data into "Schottky" and "Ohmic" regions. This distinction is discussed in detail later. Ohmic behavior is required to extract reliable carrier-transport data. Hence, mobility measurements are only plotted for the boron sheet concentration of $10^{15} \mathrm{~cm}^{-2}$ (where the $\mathrm{Si}$-III and $\mathrm{Si}$-XII pattern exhibits Ohmic behavior). All details extracted from the Hall measurements including sheet carrier concentrations and carrier type are shown in Table I.

for comparison are the resistance bar measurements. The data are divided into two behavioral regions labeled "Schottky" and "Ohmic"; this distinction will be more apparent later. Table I contains all of the Hall data, including carrier mobility, sheet carrier concentration (and type) for boron doping, together with results from initial measurements of phosphorus-doped $\mathrm{Si}$-III and Si-XII to investigate the possibility of $n$-type doping. The Hall measurements confirm the reduction in resistivity with increasing boron content observed from the resistance bar and through-tip measurements. Indeed, the resistivities measured by Hall measurements are in close agreement with those obtained from the indented bars (Fig. 4).

\section{DISCUSSION}

A highly significant result is the fact that carrier-transport measurements confirm the overall semiconducting behavior of mixed Si-III and Si-XII material (more specifically, a clear indication that $\mathrm{Si}$-XII is a semiconductor). In particular, the majority carriers are holes for boron doping and electrons for phosphorus doping (Table I). For the highest fluence boronimplanted material, where reliable mobility measurements could be made, a hole mobility (Table I) of $\sim 17 \mathrm{~cm}^{2} / \mathrm{V}$ $\mathrm{s}$ is measured. The (hole) carrier density and mobility are lower than but comparable to values expected for a similarly high (fully activated) boron concentration in single-crystal Si-I (diamond cubic silicon). ${ }^{26}$ However, compared with similarly boron doped polycrystalline $\mathrm{Si}-\mathrm{I}$, the mobility for the mixed $\mathrm{Si}$-III and Si-XII zone is higher, ${ }^{27-29}$ noting that boron can be "activated" at room temperature in our indented case but requires temperatures in excess of $600{ }^{\circ} \mathrm{C}$ in the case of conventional poly-Si-I. ${ }^{26}$ Table I summarizes the Hall data on the boron-doped material in detail. The implanted dopant appears to be electrically activated within the mixed Si-III and $\mathrm{Si}$-XII zone to an average level of $\sim 80 \%$ following indentation. Finally, for the case of phosphorus doping, the majority carriers are electrons confirming the $n$-type behavior of the Si-III and $\mathrm{Si}-\mathrm{XII}$ zone is this case. The case of phosphorus doping is considerably more complicated than boron because an $n$-doped Si-XII semiconductor will now be in contact with a hole semimetal Si-III. This may in part explain the low measured mobility for phosphorus-doped material and the comparatively higher resistivity, issues that warrant further investigation.

From available literature, Si-III and $\mathrm{Si}$-XII are believed to have differing electrical properties. Conductivity and Hall measurements of microcrystalline Si-III (created in a highpressure cell) revealed that it is a hole semimetal with $p \approx 5 \times 10^{20} \mathrm{~cm}^{-3}$ at room temperature. ${ }^{14} \mathrm{We}$ might expect that the overall electrical properties of Si-III formed by indentation should be similar to those for $\mathrm{Si}$-III created in a high-pressure cell, noting that Raman spectroscopy and TEM data on indentation-produced $\mathrm{Si}$-III and $\mathrm{Si}-\mathrm{XII}$ zones clearly identify grains with a $\mathrm{Si}$-III structure. .,7,12,30 $^{-1}$ Therefore, we suggest that this very conductive phase of Si would not be substantially altered electrically (over two orders of magnitude in conductivity) by the introduction of boron implanted to a peak concentration of $\sim 10^{20} \mathrm{~cm}^{-3}$ and lower. Less is known about the electrical properties of Si-XII because only small amounts of this phase (within a much more plentiful matrix of Si-III) have been obtained following pressure release in a diamond-anvil cell. ${ }^{1,31}$ Despite the fact that indentation can produce proportionally larger volumes of Si-XII ( $80 \%$ volume fraction, although mixed with $\mathrm{Si}-\mathrm{III}$; see the Introduction), there have been no previous studies of its electrical properties. However, $a b$ initio theoretical studies of the electronic and structural properties of Si-XII have been performed, and it is predicted that $\mathrm{Si}$-XII is either a semimetal ${ }^{16,17}$ or, more recently, a narrow-band-gap ( $\sim 240 \mathrm{meV}$ ) semiconductor. ${ }^{15,32}$ Our experimental results are

TABLE I. Summary of Hall measurements made on zones of Si-III and Si-XII formed in $a$-Si.

\begin{tabular}{|c|c|c|c|}
\hline $\begin{array}{l}\text { Matrix within which } \\
\text { Si-III/Si-XII are formed }\end{array}$ & Sheet resistivity $(\Omega / \mathrm{sq})$ & $\begin{array}{l}\text { Carrier density }\left(\mathrm{cm}^{-2}\right) \\
\text { (implanted sheet concentration of } 10^{15} \mathrm{~cm}^{-2} \text { ) }\end{array}$ & Carrier mobility $\left(\mathrm{cm}^{2} V^{-1} s^{-1}\right)$ \\
\hline$a$-Si, implanted with boron & $464 \pm 30$ & $8.1 \pm 0.9 \times 10^{14}(p$-type $)$ & $17 \pm 0.8$ \\
\hline$a-\mathrm{Si}$, boron-free & $2553 \pm 100$ & & \\
\hline$a-\mathrm{Si}$, implanted with phosphorus & $730 \pm 5$ & $2.2 \pm 0.1 \times 10^{15}(n$-type $)$ & $4.0 \pm 0.1$ \\
\hline
\end{tabular}


(a)
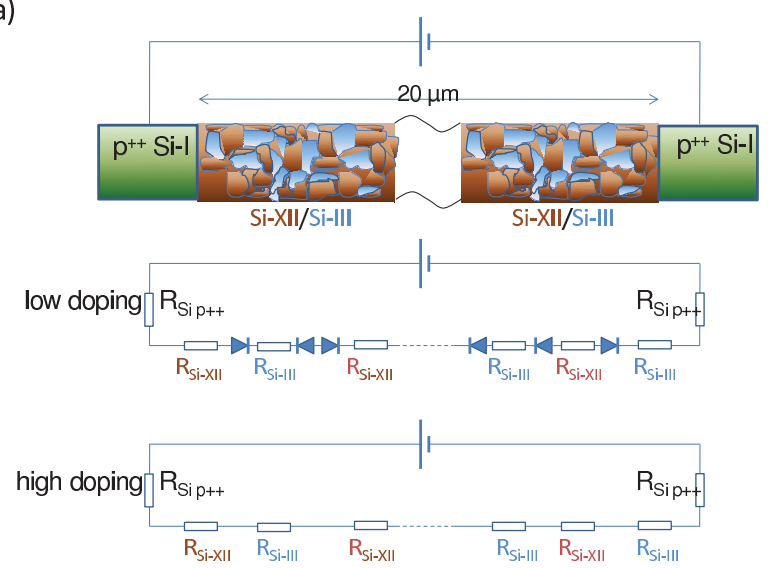

(b)

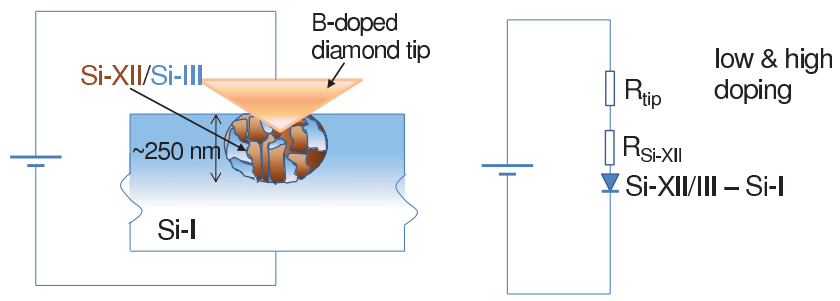

FIG. 6. (Color online) Proposed model to explain the observed electrical properties of mixed Si-XII and SI-III regions made in this study. The zones are composed of $\sim 80 \%$ of Si-XII (from Raman measurements). Despite this, for large zones (several micrometers) of the mixed phases (made by overlapping individual indents in bar and Hall measurements) current flows through a series of electrical junctions formed between grains of Si-III and Si-XII (tens of nm in dimension). For low doping levels, these electrical junctions are Schottky-like owing to the semiconductor and semimetal contact between $\mathrm{Si}$-XII and $\mathrm{Si}$-III grains. Thus the measured $\mathrm{I}-\mathrm{V}$ characteristics are a result of current flow through a series of back-to-back Schottky junctions, regardless of the polarity of the measurement. As the doping is increased, these Schottky barriers become increasingly "electrically leaky" and eventually the Schottky barrier is eliminated [see (a) and Fig. 4]. For the through-tip measurements made in this study (b), the size of the zone of Si-III and Si-XII is approximately two orders of magnitude smaller. In this case, it is highly likely that continuous paths of Si-XII can be formed through the zone in the direction of current flow, because $\mathrm{Si}$-XII is the dominant phase ( $~ 80 \%$ volume) and the direction of current flow is in the direction of grain growth. Thus, such through-wafer measurements will be dominated by conducting paths of the semiconducting Si-XII phase. Rectifying $I-V$ characteristics originate from a Schottky contact between the $\mathrm{Si}-\mathrm{XII}$ and the underlying crystalline $\mathrm{Si}-\mathrm{I}$ (diamond cubic) substrate.

consistent with this latter prediction of $\mathrm{Si}-\mathrm{XII}$ to be a semiconductor, albeit with some intriguing properties, whereby both boron and phosphorus appear to be electrically active within the lattice following indentation at room temperature without the need for postindentation annealing. We now provide a more detailed interpretation of our results that strengthens support for our claim that the semiconducting behavior of Si-XII can be inferred from our electrical results on the mixed Si-XII and Si-III phases.
We first discuss the origin of the observed "Schottky-like" and "Ohmic-like" behavior of the various structures measured here. For conduction within the $\mathrm{Si}$-III and $\mathrm{Si}-\mathrm{XII}$ zones, we suggest that the Schottky-like behavior is a result of the dominant contact properties between Si-III (semimetal) and Si-XII (semiconductor) grains. When the doping level is low and the test structure is large compared to the size of individual grains of Si-III and Si-XII (the case for the resistance bar and Hall patterns), current is driven through many reverse-biased Schottky contacts regardless of the polarity of the applied voltage. We illustrate this situation in Fig. 6(a). When the doping is high, these Schottky barriers are eliminated and the zone exhibits excellent Ohmic behavior across individual grains. Thus, for reliable resistivity, carrier density, and carrier mobility measurements, these barriers must be negligible and this requires high $\mathrm{B}$ doping. As a result, reliable mobility measurements could only be obtained for the highest boron concentration where the mixed phase material is Ohmic. A consequence of this behavior is the lack of scaling of resistivity with B concentration when the B concentrations straddle the Schottky and Ohmic regions in Fig. 5. For the through-tip measurements, where the zones of Si-III and $\mathrm{Si}$-XII material are $\sim 200 \mathrm{~nm}$ in size (for the measurements here) and the current flow is in the same direction as the grain growth, there is a large probability that continuous current paths exist that are composed entirely of Si-XII [Fig. 6(b)]. Note that the dominant phase in the mixture is $\mathrm{Si}$-XII (see earlier) and this enhances the probability of connectivity through $\mathrm{Si}$-XII grains in throughtip measurements. Thus, the through-tip characteristics are Schottky-like because the Si-III and Si-XII zones form a rectifying contact with the lightly doped underlying crystalline Si-I matrix (attributable to the difference in band gap between Si-XII and Si-I). An additional consequence of the through-tip measurements is that the resistivity under forward bias scales with boron concentration (see Fig. 3).

\section{CONCLUSION}

In summary, the electrical measurements of mixed Si-III and $\mathrm{Si}$-XII end phases formed by indentation at room temperature have shown that they are highly conducting and that the conductivity can be controlled by (room-temperature) doping with boron or phosphorus without the need for postindentation annealing. The most significant finding of this study is that the mixed Si-III and Si-XII phases exhibit semiconducting character, and our results strongly suggest that the Si-XII phase is a semiconductor, exhibiting $p$ - or $n$-type behavior when the starting amorphous material contains boron or phosphorus, respectively. Furthermore, the boron activation in $\mathrm{Si}-\mathrm{XII}$ immediately following indentation appears to be excellent and comparable with that for the single-crystalline diamond cubic Si-I phase. Indeed, following boron implantation and room-temperature indentation of $a$-Si, the measured (hole) carrier concentration (from a mixed $\mathrm{Si}$-III and $\mathrm{Si}$-XII phase of $\sim 80 \% \mathrm{Si}$-XII) approaches the boron concentration even for a very high average concentration of $\sim 10^{20} \mathrm{~cm}^{-3}$. In such a case the Hall mobility is $17 \mathrm{~cm}^{2} / \mathrm{V} \mathrm{s}$, an impressively high value compared with heavily doped poly-Si-I that has undergone thermal annealing to above $600{ }^{\circ} \mathrm{C}$. 


\section{ACKNOWLEDGMENTS}

The authors are grateful to G. Pearson for help with the Hall measurements. This work was funded by the Australian
Research Council with additional travel support from the Australian Research Network for Advanced Materials. We are also grateful to WRiota Pty. Ltd. for their partnership and involvement in this work. *simon.ruffell@anu.edu.au

${ }^{1}$ J. Crain, G. J. Ackland, J. R. Maclean, R. O. Piltz, P. D. Hatton, and G. S. Pawley, Phys. Rev. B 50, 13043 (1994).

${ }^{2}$ R.H. Wentore, Jr. and J. S. Kasper, Science 139, 338 (1963).

${ }^{3}$ S. Ruffell, B. Haberl, S. Koenig, J. E. Bradby, and J. S. Williams, J. Appl. Phys. 105, 093513 (2009).

${ }^{4}$ J. E. Bradby, J. S. Williams, J. Wong-Leung, M. V. Swain, and P. Munroe, Appl. Phys. Lett. 77, 3749 (2000).

${ }^{5}$ J. E. Bradby, J. S. Williams, J. Wong-Leung, M. V. Swain, and P. Munroe, J. Mater. Res. 16, 1500 (2001).

${ }^{6}$ D. R. Clarke, M. C. Kroll, P. D. Kirchner, R. F. Cook, and B. J. Hockey, Phys. Rev. Lett. 60, 2156 (1988).

${ }^{7}$ Y. G. Gogotsi, V. Domnich, S. N. Dub, A. Kailer, and K. G. Nickel, J. Appl. Phys. 15, 871 (2000).

${ }^{8}$ A. Kailer, Y. G. Gogotsi, and K. G. Nickel, J. Appl. Phys. 81, 3057 (1997).

${ }^{9}$ A. B. Mann, D. van Heerden, J. B. Pethica, and T. P. Weihs, J. Mater. Res. 15, 1754 (2000).

${ }^{10}$ S. Ruffell, J. E. Bradby, and J.S. Williams, Appl. Phys. Lett. 89, 091919 (2006).

${ }^{11}$ Annealing in an inert ambient at $450{ }^{\circ} \mathrm{C}$ for 30 min relaxes the ionimplanted amorphous silicon. The process repairs dangling bonds in the random network of silicon atoms. Unrelaxed amorphous silicon flows plastically and does not undergo the phase transformations.

${ }^{12}$ B. Haberl, J. E. Bradby, S. Ruffell, J. S. Williams, and P. Munroe, J. Appl. Phys. 100, 013520 (2006).

${ }^{13}$ S. Ruffell, J. E. Bradby, J. S. Williams, and P. Munroe, J. Appl. Phys. 102, 063521 (2007).

${ }^{14}$ J. M. Beeson, E. H. Mokhtari, J. Gonzalez, and G. Weill, Phys. Rev. Lett. 59, 473 (1987).

${ }^{15}$ B. D. Malone, J. D. Sau, and M. L. Cohen, Phys. Rev. B 78, 035210 (2008)
${ }^{16}$ B. G. Pfrommer, M. Cote, S. G. Louie, and M. L. Cohen, Phys. Rev. B 56, 6662 (1997).

${ }^{17}$ R. O. Piltz, J. R. Maclean, S. J. Clark, G. J. Auckland, P. D. Hatton, and J. Crain, Phys. Rev. B 52, 4072 (1995).

${ }^{18}$ S. Ruffell, J. E. Bradby, N. Fujisawa, and J. S. Williams, J. Appl. Phys. 101, 083531 (2007).

${ }^{19}$ S. Ruffell, J. E. Bradby, and J. S. Williams, J. Mater. Res. 22, 578 (2007).

${ }^{20}$ V. Domnich, Y. Gogotsi, and S. Dub, Appl. Phys. Lett. 76, 2214 (2000).

${ }^{21}$ D. Ge, A. M. Minor, E. A. Stach, and J. W. Morris Jr., Philos. Mag. 86, (25-26): 4069 (2006).

${ }^{22}$ J.-i. Jang, M. J. Lance, S. Wen, T. Y. Tsui, and G. M. Pharr, Acta Mater. 53, 1759 (2005).

${ }^{23}$ T. Juliano, V. Domnich, and Y. Gogotsi, J. Mater. Res. 19, 3099 (2004).

${ }^{24}$ G. M. Pharr, W. C. Oliver, and D. S. Harding, J. Mater. Res. 6, 1129 (1991).

${ }^{25}$ I. Zarudi, L. C. Zhang, and M. V. Swain, J. Mater. Res. 18, 758 (2003).

${ }^{26}$ W. E. Beadle, J. C. C. Tsai, and R. D. Plummer, Quick Reference Manual for Silicon Integrated Circuit Technology (Wiley, New York, 1985).

${ }^{27}$ J.Y. W. Seto, J. Appl. Phys. 46, 5247 (1975).

${ }^{28}$ D. M. Kim, F. Qian, C. U. Bickford, and H. K. Park, IEEE Trans. Electron Devices 34, 1774 (1987).

${ }^{29}$ B. Ai, H. Shen, Z. Liang, Z. Chen, G. Kong, and X. Liao, Thin Solid Films 197, 157 (2006).

${ }^{30}$ V. Domnich and Y. Gogotsi, Rev. Adv. Mater. Sci. 3, 1 (2002).

${ }^{31}$ H. Olijnyk and A.P. Jephcoat, Phys. Status Solidi B 211, 413 (1999).

${ }^{32}$ B. D. Malone, J. D. Sau, and M. L. Cohen, Phys. Rev. B 78, 161202(R) (2008). 